\title{
Effect of Fungicide and Timing of Application on Management of Sunflower Rust
}

\author{
Andrew J. Friskop, Department of Plant Pathology, North Dakota State University, Fargo 58102; Thomas J. Gulya, USDA-ARS Northern \\ Crop Science Laboratory, Fargo, ND 58108; Scott A. Halley, North Dakota State University, Langdon Research Extension Center, Langdon \\ 58249; Blaine G. Schatz, North Dakota State University, Carrington Research Extension Center, Carrington 58421; Joel P. Schaefer, CHS \\ Sunflower, Grandin, ND 58038; James G. Jordahl and Scott M. Meyer, Department of Plant Pathology, North Dakota State University, Fargo \\ 58102; Kevin W. Misek, North Dakota State University, Langdon Research Extension Center, Langdon 58249; Paul Hendrickson, North \\ Dakota State University, Carrington Research Extension Center, Carrington 58421; and Samuel G. Markell, Department of Plant Pathology, \\ North Dakota State University, Fargo 58102
}

\begin{abstract}
Friskop, A. J., Gulya, T. J., Halley, S. A., Schatz, B. G., Schaefer, J. P., Jordahl, J. G., Meyer, S. M., Misek, K. W., Hendrickson, P., and Markell, S. G. 2015. Effect of fungicide and timing of application on management of sunflower rust. Plant Dis. 99:1210-1215.

Sunflower rust is an important yield-limiting disease in sunflower production in the Great Plains of the United States. Rust severity and incidence have increased between 2002 and 2011, and genetic resistance is limited in most commercial hybrids, particularly the high-value confectionary market type. Although fungicides are available for rust management in the United States, management recommendations are insufficient. Specifically, efficacy and timing data are very limited for fungicides in FRAC groups 7 and 11 . Seventeen fungicide efficacy and timing trials were conducted between 2008 and 2011 in North Dakota. Timings evaluated

across the four years included single or multiple applications at growth stages (GS): GS V8-V12 (late vegetative), GS R1 (terminal bud formation), GS R3-4 (elongation of bud), GS R5 (flowering), and GS R6 (completion of flowering). With few exceptions, fungicide applications of DMIs and QoIs controlled disease greater than SDHI fungicides. Fungicide applications made at R5, either singly or in combination, consistently resulted in greater disease control. A negative correlation $(r=-0.7756)$ between disease control and yield was observed, resulting in a yield reduction of $6.6 \%$ for every $1 \%$ increase in disease severity.
\end{abstract}

Sunflower rust, caused by Puccinia helianthi Schwein., is an important yield-limiting disease in United States sunflower production areas. The pathogen is macrocyclic, autoecious, and can overwinter in all areas of sunflower production in the United States (Gulya et al. 1997; Harveson 2010; Markell et al. 2009b). Consequently, the pathogen has potential for frequent sexual reproduction, which can result in race changes. Similarly, earlier onset of rust in producers' fields can lead to the development of greater year-end severity levels and yield loss. Sunflower rust incidence has increased in North Dakota since 2008 and yield losses of up to $80 \%$ have been documented (Kandel 2012). Most commercially grown hybrids are susceptible to common races of rust (Friskop et al. 2011a), and the impact of crop rotation on rust is limited because of long-distance spore dispersal and ubiquitous wild sunflower populations harboring the pathogen, leaving fungicides as one of the only effective management tools.

The majority of the studies evaluating the performance of fungicides for sunflower rust management were conducted outside the United States. Results from efficacy trials conducted in Kenya and Turkey demonstrated that fungicides increased yield-component values when compared with nontreated controls (Singh 1975; Thakore et al. 1980). In the 1990s in Israel, Shtienberg (1995) developed a fungicide action threshold based on both leaf severity and growth stage and recommended a fungicide application when leaf severity of $3 \%$ on the upper four leaves is reached prior to 27 days after flowering. However, development of the threshold was based solely on research conducted with demethylation inhibitors, and in a production system different from the north-central United States (humidity, day length, and temperature). Since then, quinone outside inhibitors have been

\section{Corresponding Author S. G. Markell, Email: samuel.markell@ndsu.edu}

*The $\boldsymbol{e}$-Xtra logo stands for "electronic extra" and indicates that 2 supplementary figures are included in the online edition.

Accepted for publication 21 November 2014.

http://dx.doi.org/10.1094/PDIS-10-14-1036-RE

(C) 2015 The American Phytopathological Society labeled (McMullen and Markell 2010), and are recommended for management of other rust pathosystems (Mueller et al. 2009; Olson et al. 2011; Wanyera et al. 2009), but efficacy and timing for sunflower rust has not been investigated. The objectives of this study were to evaluate fungicide efficacy and timing for management of sunflower rust and protection of yield.

\section{Materials and Methods}

Locations and experimental design. Experiment sites were at four locations in North Dakota: Casselton (CASS), the NDSU Carrington Research Extension Center (CREC), Grandin (GRAN), and the NDSU Langdon Research Extension Center (LREC). Trials were conducted in a randomized complete block design with four replications at CASS in 2008, CREC from 2008 to 2011, GRAN from 2009 to 2011, and LREC from 2008 to 2011. CREC was under centerpivot irrigation, all other locations were nonirrigated. Four row plots were seeded with a susceptible confection hybrid 'Jaguar' (Seeds2000, Breckenridge, MN). Row width was $76.2 \mathrm{~cm}$ and seed spacing was $21.6 \mathrm{~cm}$ at each location. Four row plots were used at all locations; the middle two rows of each plot were used for data collection, while the other plot rows were used as borders to interplot interference. Planting date, rust detection date, and plot length varied at each location (Table 1). Agronomic practices appropriate for sunflower production were followed for each location and year (Berglund 2007).

Artificial inoculation. In order to facilitate disease development, plots were artificially inoculated at each location. Inoculum was produced by increasing urediniospores of $P$. helianthi isolate ND07-01 (race 336) on a greenhouse-grown susceptible sunflower hybrid to ensure a fresh viable source of inoculum. Collected spores were suspended in Soltrol 170 and quantified to approximately 275,000 spores $/ \mathrm{ml}$. The spore suspension was applied using a backpack mounted leaf blower (model \# BR600, Stihl Incorporated USA, Virginia Beach, VA) with small modifications to the air wand (Markell et al. 2009a). A $0.5 \mathrm{~cm}$ diameter hole was drilled approximately $13.7 \mathrm{~cm}$ from the mouth of the wand, on the top side. A $0.5 \mathrm{~cm}$ outside-diameter hose was inserted approximately $1 \mathrm{~cm}$ into the hole in the wand and secured with adhesive tape (item \# 283227, ShurTech Brands, Avon, $\mathrm{OH}$ ). The urediniospore suspension was injected 
through the hose and into the airstream where it vaporized and was dispersed onto plant tissue. In 2008 and 2009, only border rows of the plots and internal spreaders plots were inoculated, while in 2010 and 2011, the treatment rows were inoculated. Timing of inoculation coincided with disease onset objectives. Inoculation was completed during early sunflower reproductive stages in 2008 and 2009; and in late vegetative stages during 2010 and 2011.

Fungicide efficacy. Efficacy trials were established in 2008 and 2009. Locations varied in the total number of fungicides evaluated but all trials included the demethylation inhibitors (DMI - FRAC 3); prothioconazole (Proline, Bayer CropScience, Research Triangle Park, NC), prothioconazole + tebuconazole (Prosaro, Bayer CropScience), tebuconazole (Tebuzol, United Phosphorous Inc., King of Prussia, PA), and the quinone outside inhibitors (QoI FRAC 11); pyraclostrobin (Headline, BASF, Research Triangle Park, NC) and azoxystrobin (Quadris, Syngenta Crop Protection, Greensboro, NC). Other DMI fungicides included at some locations were metconazole (Quash, Valent USA, Walnut Creek, CA) and tebuconazole (Folicur, Bayer CropScience) (Table 1). Succinate dehydrogenase inhibitors (SDHI - FRAC 7) included at some locations were boscalid (Endura, BASF) and penthiopyrad (Vertisan, DuPont Agricultural Products, Wilmington, DE). Additionally, a nontreated control (NTC) was used at each location. All fungicides were applied between growth stages R5.0 (start of flowering) and R5.5 (midflowering) (Schneiter et al. 1998).

Fungicide timing-Normal onset. Timing trials conducted in 2008 and 2009 were designed to simulate the onset of rust in a "normal" disease onset scenario. We define a normal disease onset as when rust is first found on the upper leaves of sunflowers during the reproductive stages of development. This approximately simulates the time of disease onset in production fields when the source of inoculum are urediniospores from nearby fields or long distance dispersal. To simulate this, spreader rows were inoculated with urediniospores approximately 1 to 2 weeks before the reproductive growth stages began in the trials. Fungicide applications were made at sunflower growth stages (Schneiter et al. 1998) R3-4 (midbud elongation), R5 (flowering), and R6 (flowering complete) using single or multiple applications of pyraclostrobin or tebuconazole. An NTC was used at each location as well. The number of treatments varied among location and years (Table 1).

Fungicide timing-Early onset. Timing trials conducted in 2010 and 2011 were designed to simulate an "early" disease onset scenario. We define early disease onset as when rust is found on the upper portion of the plant prior to growth stage $\mathrm{R} 1$. This approximately simulates the time of disease onset in production fields when the source of inoculum originates from within the field. To simulate this, all plots were initially inoculated with urediniospores between V6 and V12 growth stages, and reinoculated periodically until uredinia were visually observed. Fungicide applications were made at three growth stages (Schneiter et al. 1998): V8-V12 (late vegetative), R1 (bud formation), and R5 (bloom) using single and multiple applications. Experimental timings were developed, in part, to coincide with sunflower production practices. Herbicide applications are usually initiated at late vegetative (V8-V12), fungicide applications for nondisease yield impacts have been suggested to be applied at R1, and the R5 timing was deemed the most important fungicide timing of the three tested in 2008 and 2009. Pyraclostrobin was used at all locations, while tebuconazole was also evaluated at GRAN (Table 1).

Fungicide application. Fungicides were applied at $241.3 \mathrm{kPa}$ with $\mathrm{CO}_{2}$ powered backpack sprayers at GRAN, CASS, and LREC and with a tractor sprayer at CREC. Applications were made at 187 liters/ha at GRAN and CASS, 86 liters/ha at LREC, and 121.6 liters/ha at CREC. Teejet 8002 flat fan nozzles were used at all locations. Nozzle spacing was $76.2 \mathrm{~cm}$ at CASS, GRAN, and LREC and $38.1 \mathrm{~cm}$ at CREC. The target area of application was the upper four leaves of the sunflower plant.

Data collection. Disease evaluations were conducted visually, with the aid of rust assessment diagrams (Fig. 1) (Friskop et al. 2011a; Gulya et al. 1990). The sunflower rust assessment diagrams are more conservative than other rust assessment tools. For example, a $1 \%$ rust severity on sunflower rust diagrams translates to approximately a $20 \%$ severity for wheat rust using the modified Cobb scale (Peterson et al. 1948). The mean percent rust severity on the upper four leaves of 10 randomly selected plants in the center two rows of each plot were evaluated (Shtienberg 1995). Assessments were conducted at approximately 2 week intervals beginning at disease onset and ending at plant maturity (R8-R9). Disease severity ratings were used to calculate the area under disease progress curve (AUDPC) values and relative area under disease progress curve (rAUDPC) (Madden et al. 2007). AUDPC was calculated as:

$$
\operatorname{AUDPC}=\sum_{i=1}^{n}\left(\left(y_{i}+y_{i+1} / 2\right)\left(t_{i+1}-t_{i}\right)\right)
$$

where $y_{i}=$ rust severity at the $i$-th observation, $t_{i}=$ time (days) at the $i$-th observation, and $n=$ total number of observations. The rAUDPC was calculated as:

$$
\mathrm{rAUDPC}=\mathrm{AUDPC} /\left(t_{f}-t_{0}\right) \times 100
$$

where $t_{f}=$ the duration of days at the final rating and $t_{0}=$ the time of disease onset. Percent disease control (hereafter referred to as disease

\begin{tabular}{|c|c|c|c|c|c|c|c|c|c|}
\hline Location & Planting date & Trial type & $\begin{array}{l}\text { Row } \\
\text { length } \\
(\mathbf{m})\end{array}$ & $\begin{array}{c}\text { Row } \\
\text { width } \\
(\mathbf{c m})\end{array}$ & $\begin{array}{c}\text { Seed } \\
\text { spacing } \\
(\mathrm{cm})\end{array}$ & GS $^{\mathrm{a}}$ application & $\begin{array}{c}\text { Number } \\
\text { of } \\
\text { fungicides }\end{array}$ & $\begin{array}{c}\text { Rust } \\
\text { detection } \\
\text { date }\end{array}$ & $\begin{array}{c}\text { Yield } \\
\text { obtained }\end{array}$ \\
\hline Casselton, ND & 19 Jun 08 & Efficacy & 7.6 & 76.2 & 21.6 & $\mathrm{R} 5$ & 7 & 21 Aug 08 & Yes \\
\hline Casselton, ND & 19 Jun 08 & Timing-Normal onset & 7.6 & 76.2 & 21.6 & R3, R5, R6 & 2 & 21 Aug 08 & Yes \\
\hline Carrington, ND & 22 May 08 & Efficacy & 7.6 & 76.2 & 21.6 & R5 & 8 & 7 Aug 08 & Yes \\
\hline Carrington, ND & 22 May 08 & Timing-Normal onset & 7.6 & 76.2 & 21.6 & R3, R5, R6 & 1 & 7 Aug 08 & Yes \\
\hline Langdon, ND & 20 May 08 & Efficacy & 4.6 & 76.2 & 21.6 & R5 & 8 & 7 Aug 08 & Yes \\
\hline Langdon, ND & 20 May 08 & Timing_-Normal onset & 4.6 & 76.2 & 21.6 & R3, R5, R6 & 2 & 7 Aug 08 & Yes \\
\hline Carrington, ND & 1 Jun 09 & Efficacy & 7.6 & 76.2 & 21.6 & R5 & 5 & $30 \mathrm{Jul} 09$ & Yes \\
\hline Carrington, ND & 1 Jun 09 & Timing-Normal onset & 7.6 & 76.2 & 21.6 & R3, R5, R6 & 2 & $30 \mathrm{Jul} 09$ & Yes \\
\hline Grandin, ND & 1 Jun 09 & Efficacy & 7.6 & 76.2 & 21.6 & R5 & 10 & $30 \mathrm{Jul} 09$ & No \\
\hline Grandin, ND & 1 Jun 09 & Timing-Normal onset & 7.6 & 76.2 & 21.6 & R3, R5, R6 & 2 & $30 \mathrm{Jul} 09$ & No \\
\hline Langdon, ND & 11 May 09 & Efficacy & 4.6 & 76.2 & 21.6 & R5 & 7 & $21 \mathrm{Jul} 09$ & Yes \\
\hline Langdon, ND & 11 May 09 & Timing-Normal onset & 4.6 & 76.2 & 21.6 & R3, R5, R6 & 2 & $21 \mathrm{Jul} 09$ & Yes \\
\hline Carrington, ND & 27 May 10 & Timing_Early onset & 7.6 & 76.2 & 21.6 & V8-V12, R1, R5 & 1 & 7 Jul 10 & No \\
\hline Grandin, ND & 28 May 10 & Timing_Early onset & 7.6 & 76.2 & 21.6 & V8-V12, R1, R5 & 2 & $28 \mathrm{Jul} 10$ & No \\
\hline Langdon, ND & 19 May 10 & Timing_Early onset & 4.6 & 76.2 & 21.6 & V8-V12, R1, R5 & 1 & 7 Jul 10 & Yes \\
\hline Grandin, ND & 25 May 11 & Timing_Early onset & 7.6 & 76.2 & 21.6 & V8-V12, R1, R5 & 2 & 22 Jul 11 & Yes \\
\hline Langdon, ND & 30 May 11 & Timing_Early onset & 4.6 & 76.2 & 21.6 & V8-V12, R1, R5 & 1 & $11 \mathrm{Jul} 11$ & Yes \\
\hline
\end{tabular}

Table 1. Agronomic and trial information for all experimental locations

\footnotetext{
a Sunflower growth stages according to Schneiter et al. (1998).
} 
control) was calculated at each location using the formula ((1 $($ rAUDPC of a treatment / rAUDPC of the NTC) $\times 100)$. Yield was collected from the center two plot rows at plant maturity. Yield loss assessment was evaluated by correlating R7 leaf severities to percent change in yield from the nontreated control. Only trials where observed rust severity on the NTC was greater than 5\% at R7 and where significant yield differences occurred were used for yield loss assessment. Namely, CREC 2008 Timing, CREC 2009 Efficacy, CREC 2009 Timing, and LREC 2010.

Statistical analysis. Data were analyzed at each location separately due to differences in the establishment and development of
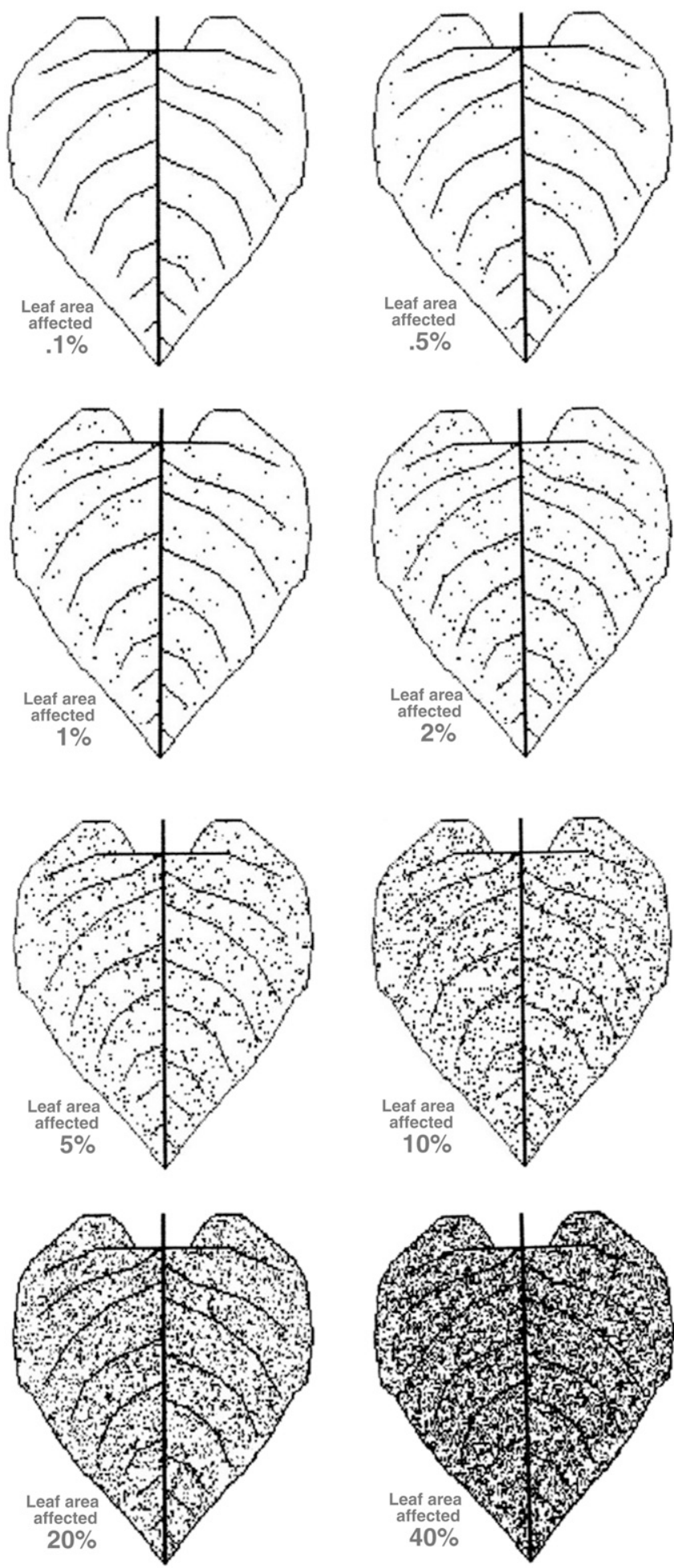

Fig. 1. Sunflower rust severity assessment diagrams. Reproduced from Friskop et al. (2011a). disease in the trials. Analysis of variance (ANOVA) was used in the general linear models procedure within the SAS 9.2 program (SAS Institute, Cary, NC). Fisher's protected least significant differences at $\alpha=0.05$ were used to determine significance difference among treatment means. Pearson's correlation coefficient and simple linear regression procedure within the SAS 9.2 program was used to assess yield loss.

\section{Results}

Fungicide efficacy. Rust developed at all locations in 2008 , and R7 severity was high enough on the NTC at CREC 2008 and CASS 2008 to see differences in disease control among treatments (Table 2). Due to the low amount of disease pressure at LREC 2008, statistically significant levels of disease control were not found among the fungicides tested and numerically negative disease control values were an artifact of very low disease pressure. At CREC 2008 and CASS 2008, disease control provided by DMI fungicides was statistically higher than that of the only SDHI tested at that location, penthiopyrad. At CREC 2008, statistical differences between the QoI treatments pyraclostrobin and azoxystrobin were observed. At CASS 2008 , rust severity at R5 and R7 was relatively low, but year-end severities were among the highest at physiological maturity (data not shown).

In 2009, rust developed at all locations with great enough severity that disease control differences among treatments were observed (Table 2). DMI fungicide applications resulted in greater levels of disease control than a boscalid application at locations where it was tested. Greater disease control was achieved with tebuconazole and prothioconazole + tebuconazole applications than azoxystrobin at CREC and GRAN in 2009. At LREC 2009, levels of disease control did not significantly differ between applications of pyraclostrobin and the DMI fungicides tested. At CREC 2009, applications of DMIs and pyraclostrobin resulted in a statistically greater yield than the NTC. However, yield between the azoxystrobin treatment and the NTC was the same.

Fungicide timing-Normal onset. Rust developed at all locations in 2008, but severity was great enough only at CASS 2008 and CREC 2008 to observe meaningful differences in disease control. Yield differences were observed only at CREC 2008 (Table 3). At CASS 2008, disease control from fungicide applications ranged from 97.3 to $71.6 \%$, and statistical differences were observed among treatments. Yield was not statistically different than the NTC for any fungicide application. At CREC 2008, significant differences in disease control and yield were observed among fungicide timings. Levels of disease control and yield were the same between a single application of pyraclostrobin at R5 and three fungicide applications of pyraclostrobin, both of which were statistically different than the NTC.

Rust developed at all locations in 2009 , and severity was great enough to see meaningful statistical difference in disease control (Table 3). In CREC 2009 and LREC 2009, disease control with an R5 application of pyraclostrobin or tebuconazole was statistically the same as three applications of fungicides. Statistical differences in disease control were observed among single application timings in both trials. Five fungicide treatments in CREC 2009 resulted in statistically higher yield than the NTC, and differences in yield among treatments was observed. At GRAN 2009, rust was not effectively controlled by singular applications made at R6.

Fungicide timing-Early onset. An early disease onset was not achieved at any locations in 2010; however, rust severities were high enough to observe significant differences among treatments (Table 4). At CREC 2010, disease control was significantly higher than the NTC when single applications of pyraclostrobin were made. Also, disease control levels were the same with pyraclostrobin applications made at R1, R5, and V8-V12 + R1 + R5. No differences were observed among treatments made up of multiple applications of pyraclostrobin. At GRAN 2010, disease control from single fungicide applications at V8-V12 and R1 was statistically the same as the NTC. At LREC 2010, disease control levels of four fungicide treatments were significantly different 
than the NTC. Disease control values were among the highest for treatments containing multiple applications of pyraclostrobin when a R5 timing application was included. However, yield differences were not observed among treatments with multiple applications.

An early disease onset in 2011 did not occur at any location. However, significant differences in disease control were observed among treatments (Table 4). At GRAN 2011, disease control was greater than the NTC for all applications except a single application at V8-V12 of pyraclostrobin. The highest levels of disease control were achieved with multiple applications of a fungicide, which often included a R5 application. At LREC 2011, disease control was significantly higher for all fungicide applications than the NTC. Levels of disease control and yield were statistically the same among multiple pyraclostrobin applications at V8-V12 and R1, R1 and R5, and at all three timings.
Yield loss assessment. To determine the impact of rust on yield, trials CREC 2008-Normal Onset, CREC 2009 Efficacy, CREC 2009-Normal Onset, and LREC 2010 were selected for analysis because R7 severity values on the NTC were greater than $5 \%$ and significant differences were observed in yields. A negative correlation of $r=-0.7756$ was found between percent change in yield from the NTC and fungicide treatment at R7 severity values (Fig. 2). Specifically, for every $1 \%$ increase in severity at R7, yields decreased by $6.6 \%$.

\section{Discussion}

Results from this study demonstrated that fungicide applications can reduce sunflower rust severity, and with few exceptions, DMIs and QoIs reduced disease greater than the SDHIs tested in this study. Results also demonstrated management of rust depended greatly on timing, and an R5 application, which typically corresponded to

Table 2. Sunflower rust severity, percent disease control, and yield for fungicide efficacy trials in 2008 and 2009

\begin{tabular}{|c|c|c|c|c|c|c|c|c|c|c|c|c|c|c|c|c|c|c|c|c|c|c|c|c|c|}
\hline & & \multicolumn{4}{|c|}{ CASS 2008} & \multicolumn{4}{|c|}{ CREC 2008} & \multicolumn{4}{|c|}{ LREC 2008} & \multicolumn{4}{|c|}{ CREC 2009} & \multicolumn{4}{|c|}{ GRAN 2009} & \multicolumn{4}{|c|}{ LREC 2009} \\
\hline \multicolumn{2}{|c|}{ Treatments } & $\begin{array}{c}\text { R5 } \\
\text { Sev }^{c}\end{array}$ & $\begin{array}{c}\text { R7 } \\
\text { Sev } \\
\%\end{array}$ & $\begin{array}{c}\% \\
\text { Dis } \\
\text { Cont }^{\text {d }}\end{array}$ & $\begin{array}{l}\text { Yld } \\
\text { (kg/ } \\
\text { ha) }\end{array}$ & $\begin{array}{c}\text { R5 } \\
\text { Sev } \\
\%\end{array}$ & $\begin{array}{c}\text { R7 } \\
\text { Sev } \\
\%\end{array}$ & $\begin{array}{c}\% \\
\text { Dis } \\
\text { Cont }\end{array}$ & $\begin{array}{l}\text { Yld } \\
\text { (kg/ } \\
\text { ha) }\end{array}$ & $\begin{array}{c}\text { R5 } \\
\text { Sev } \\
\%\end{array}$ & $\begin{array}{c}\text { R7 } \\
\text { Sev \% }\end{array}$ & $\begin{array}{c}\% \\
\text { Dis } \\
\text { Cont }\end{array}$ & $\begin{array}{l}\text { Yld } \\
\text { (kg/ } \\
\text { ha) }\end{array}$ & $\begin{array}{c}\text { R5 } \\
\text { Sev } \\
\%\end{array}$ & $\begin{array}{c}\text { R7 } \\
\text { Sev } \\
\%\end{array}$ & $\begin{array}{c}\% \\
\text { Dis } \\
\text { Cont }\end{array}$ & $\begin{array}{l}\text { Yld } \\
\text { (kg/ } \\
\text { ha) }\end{array}$ & $\begin{array}{c}\text { R5 } \\
\text { Sev } \\
\%\end{array}$ & $\begin{array}{c}\text { R7 } \\
\text { Sev } \\
\%\end{array}$ & $\begin{array}{c}\% \\
\text { Dis } \\
\text { Cont }\end{array}$ & $\begin{array}{l}\text { Yld } \\
\text { (kg/ } \\
\text { ha) }\end{array}$ & $\begin{array}{c}\text { R5 } \\
\text { Sev } \\
\%\end{array}$ & $\begin{array}{c}\text { R7 } \\
\text { Sev } \\
\%\end{array}$ & $\begin{array}{c}\% \\
\text { Dis } \\
\text { Cont }\end{array}$ & $\begin{array}{l}\text { Yld } \\
\text { (kg/ } \\
\text { ha) }\end{array}$ \\
\hline NTC & $\ldots$ & 0 & 1.1 & 0 & 2,245 & 2.6 & 9.9 & 0 & 2,558 & 0.3 & 0.2 & 0 & 1,715 & 0.2 & 6.9 & 0 & 1,742 & 0 & 1.5 & 0 & $e^{e}$ & 0.1 & 16.8 & 0 & 1,817 \\
\hline PRO & R5 & 0 & 0.2 & 84 & 2,005 & 0.4 & 0.5 & 94 & 2,765 & 0.3 & 1.3 & -224 & 2,271 & 0.2 & 1.2 & 65 & 2,375 & 0 & 0.2 & 86 & $\ldots$ & 0 & 3.9 & 76 & 2,066 \\
\hline TEB & R5 & 0 & 0.3 & 86 & 2,344 & 0.4 & 0.7 & 93 & 2,780 & 0.1 & 0.4 & -29 & 2,042 & 0.2 & 1.1 & 80 & 2,372 & 0 & 0.3 & 82 & $\ldots$ & 0 & 5.2 & 68 & 1,895 \\
\hline MET & R5 & $\ldots$ & $\ldots$ & $\ldots$ & $\cdots$ & 0.8 & 1.2 & 85 & 3,003 & $\cdots$ & $\cdots$ & $\ldots$ & $\cdots$ & $\cdots$ & $\cdots$ & $\cdots$ & $\cdots$ & 0 & 0.1 & 91 & $\ldots$ & 0 & 3.7 & 74 & 2,022 \\
\hline PRO+TEB & R5 & 0 & 0.3 & 91 & 2,070 & 0.4 & 0.5 & 92 & 2,541 & 0.2 & 0.4 & -29 & 1,890 & 0.2 & 1.1 & 78 & 2,484 & 0 & 0.1 & 89 & $\ldots$ & 0 & 3.9 & 76 & 2,115 \\
\hline PYR & R5 & 0 & 0.3 & 66 & 2,144 & 0.7 & 2.2 & 74 & 2,765 & 0.1 & 0.3 & -3 & 1,895 & 0.2 & 0.9 & 52 & 2,333 & 0 & 0.5 & 58 & $\ldots$ & 0.1 & 5.6 & 66 & 1,661 \\
\hline $\mathrm{AZO}$ & R5 & 0 & 0.4 & 53 & 2,268 & 1.2 & 4.6 & 44 & 2,621 & 0.1 & 0.5 & -30 & 1,920 & 0.2 & 2.0 & 37 & 2,043 & 0 & 0.4 & 48 & $\ldots$ & 0.1 & 7.4 & 58 & 1,910 \\
\hline BOS & R5 & $\ldots$ & $\ldots$ & $\ldots$ & $\ldots$ & $\ldots$ & $\ldots$ & $\ldots$ & $\ldots$ & $\ldots$ & $\ldots$ & $\ldots$ & $\ldots$ & $\ldots$ & $\ldots$ & $\ldots$ & $\ldots$ & 0 & 0.9 & 51 & $\ldots$ & 0.1 & 9.7 & 42 & 1,798 \\
\hline PEN & R5 & 0 & 0.4 & 44 & 2,215 & 1.0 & 5.4 & 44 & 2,768 & 0.2 & 0.5 & -47 & 1,988 & $\ldots$ & $\ldots$ & $\ldots$ & $\ldots$ & $\ldots$ & $\ldots$ & & $\ldots$ & $\ldots$ & $\ldots$ & $\ldots$ & $\ldots$ \\
\hline $\operatorname{LSD}(P=0$ & & ns & 0.4 & 18 & $\mathrm{~ns}$ & 0.8 & 2.1 & 19 & $\mathrm{~ns}$ & 0.1 & $\mathrm{~ns}$ & ns & $\mathrm{ns}$ & $\mathrm{ns}$ & 0.9 & 28 & 316 & $\mathrm{~ns}$ & 0.6 & 24 & $\cdots$ & ns & 4.2 & 20 & ns \\
\hline
\end{tabular}

a Fungicides tested at each location: NTC = nontreated control, PRO = prothioconazole (Proline, Bayer CropScience), TEB = tebuconazole (Tebuzol, United Phosphorus Inc.), MET = metconazole (Quash, Valent), PRO + TEB = prothioconazole + tebuconazole (Prosaro, Bayer CropScience), PYR = pyraclostrobin (Headline, BASF), AZO = azoxystrobin (Quadris, Syngenta Crop Protection), BOS = boscalid (Endura, BASF), and PEN = penthiopyrad (Vertisand, DuPont).

b Timing of fungicide application according to sunflower growth stages defined by Schneiter et al. (1998).

c $\%$ Sev $=$ mean severity on upper four leaves of sunflower using assessment diagrams (Friskop et al. 2011a).

$\mathrm{d} \%$ Disease control $=[(1-(\mathrm{rAUDPC}$ of a treatment $/ \mathrm{rAUDPC}$ of the NTC $)) \times 100] ;$ where $\mathrm{rAUDPC}=\mathrm{AUDPC} /\left(t_{f}-t_{0}\right) \times 100, \mathrm{AUDPC}=\sum_{i=1}^{n}\left(\left(y_{i}+y_{i+1} / 2\right)\left(t_{i+1}-t_{i}\right)\right)$ where $y_{i}=$ rust severity at the $i$-th observation, $t_{i}=$ time (days) at the $i$-th observation, and $n=$ total number of observations, as adapted from Madden et al. (2007).

e Yield was not obtained at GRAN 2009 due to high winds and severe lodging prior to harvest.

Table 3. Sunflower rust severity, percent disease control, and yield for normal onset timing trials in 2008 and 2009

\begin{tabular}{|c|c|c|c|c|c|c|c|c|c|c|c|c|c|c|c|c|c|c|c|c|c|c|c|c|c|}
\hline & & \multicolumn{4}{|c|}{ CASS 2008} & \multicolumn{4}{|c|}{ CREC 2008} & \multicolumn{4}{|c|}{ LREC 2008} & \multicolumn{4}{|c|}{ CREC 2009} & \multicolumn{4}{|c|}{ GRAN 2009} & \multicolumn{4}{|c|}{ LREC 2009} \\
\hline \multicolumn{2}{|c|}{ Treatments } & \multirow{2}{*}{$\begin{array}{l}\text { R5 } \\
\text { Sev } \\
\% \text { c }\end{array}$} & \multirow{2}{*}{$\begin{array}{c}\text { R7 } \\
\text { Sev } \\
\%\end{array}$} & \multirow{2}{*}{$\begin{array}{c}\% \\
\text { Dis } \\
\text { Cont }^{\text {d }}\end{array}$} & \multirow{2}{*}{$\begin{array}{l}\text { Yld } \\
\text { (kg/ } \\
\text { ha) }\end{array}$} & \multirow{2}{*}{$\begin{array}{c}\text { R5 } \\
\text { Sev } \\
\% \\
\end{array}$} & \multirow{2}{*}{$\begin{array}{c}\text { R7 } \\
\text { Sev } \\
\% \\
\end{array}$} & \multirow{2}{*}{$\begin{array}{c}\% \\
\text { Dis } \\
\text { Cont }\end{array}$} & \multirow{2}{*}{$\begin{array}{l}\text { Yld } \\
\text { (kg/ } \\
\text { ha) }\end{array}$} & \multirow{2}{*}{$\begin{array}{c}\text { R5 } \\
\text { Sev } \\
\% \\
\end{array}$} & \multirow{2}{*}{$\begin{array}{c}\text { R7 } \\
\text { Sev } \\
\% \\
\end{array}$} & \multirow{2}{*}{$\begin{array}{c}\% \\
\text { Dis } \\
\text { Cont } \\
\end{array}$} & \multirow{2}{*}{$\begin{array}{l}\text { Yld } \\
\text { (kg/ } \\
\text { ha) }\end{array}$} & \multirow{2}{*}{$\begin{array}{c}\text { R5 } \\
\text { Sev } \\
\%\end{array}$} & \multirow{2}{*}{$\begin{array}{c}\text { R7 } \\
\text { Sev } \\
\% \\
\end{array}$} & \multirow{2}{*}{$\begin{array}{c}\% \\
\text { Dis } \\
\text { Cont } \\
\end{array}$} & \multirow{2}{*}{$\begin{array}{l}\text { Yld } \\
\text { (kg/ } \\
\text { ha) }\end{array}$} & \multirow{2}{*}{$\begin{array}{c}\text { R5 } \\
\text { Sev } \\
\% \\
\end{array}$} & \multirow{2}{*}{$\begin{array}{c}\text { R7 } \\
\text { Sev } \\
\% \\
\end{array}$} & \multirow{2}{*}{$\begin{array}{c}\% \\
\text { Dis } \\
\text { Cont }\end{array}$} & \multirow{2}{*}{$\begin{array}{l}\text { Yld } \\
\text { (kg/ } \\
\text { ha) }\end{array}$} & \multirow{2}{*}{$\begin{array}{c}\text { R5 } \\
\text { Sev } \\
\% \\
\end{array}$} & R7 & $\%$ & Yld \\
\hline Fung ${ }^{a}$ & $\mathbf{A p p} \mathbf{b}^{\mathbf{b}}$ & & & & & & & & & & & & & & & & & & & & & & $\%$ & Cont & ha) \\
\hline NTC & $\ldots$ & 0 & 1.5 & 0 & 2,505 & 1.8 & 8.7 & 0.0 & 1,613 & 0.4 & 0.4 & 0 & 1,168 & 1.2 & 12.0 & 0 & 1,729 & 0 & 0.6 & 0 & $\ldots e$ & 0.4 & 22.1 & 0 & 1,167 \\
\hline PYR & $\mathrm{R} 3$ & 0 & 0.0 & 96 & 2,570 & 0.8 & 4.1 & 35 & 1,849 & 0.5 & 0.3 & -23 & 1,235 & 0.4 & 5.0 & 45 & 2,225 & 0 & 0.2 & 35 & $\ldots$ & 0.1 & 12.8 & 28 & 1,754 \\
\hline PYR & R5 & 0 & 0.8 & 72 & 2,563 & 0.8 & 1.3 & 79 & 2,042 & 0.1 & 0.2 & 46 & 1,218 & 0.9 & 1.1 & 70 & 2,571 & 0 & 0.2 & 49 & $\ldots$ & 0.0 & 4.1 & 79 & 1,729 \\
\hline PYR & R6 & 0 & 0.5 & 86 & 2,426 & 2.7 & 6.0 & 43 & 1,549 & 0.3 & 0.4 & -1 & 988 & 0.9 & 5.9 & 51 & 2,006 & 0 & 0.8 & -14 & $\ldots$ & 0.1 & 8.4 & 57 & 1,969 \\
\hline TEB & R3 & 0 & 0.3 & 79 & 2,758 & $\ldots$ & $\ldots$ & $\ldots$ & $\ldots$ & 0.1 & 0.3 & 23 & 1,260 & 0.7 & 6.9 & 35 & 1,888 & 0 & 0.0 & 87 & $\ldots$ & 0.1 & 6.9 & 66 & 1,475 \\
\hline TEB & R5 & 0 & 0.5 & 89 & 2,670 & $\ldots$ & $\ldots$ & $\ldots$ & $\ldots$ & 0.3 & 0.2 & 20 & 989 & 0.9 & 2.2 & 76 & 2,387 & 0 & 0.2 & 52 & $\ldots$ & 0.3 & 5.7 & 60 & 1,905 \\
\hline TEB & R6 & 0 & 0.6 & 92 & 2,405 & $\ldots$ & $\ldots$ & $\ldots$ & $\ldots$ & 0.3 & 0.2 & 17 & 969 & 1.0 & 7.2 & 51 & 1,767 & 0 & 0.7 & -17 & $\ldots$ & 0.1 & 9.3 & 41 & 1,588 \\
\hline PYR & $\mathrm{R} 3$ & & & & & & & & & & & & & & & & & & & & & & & & \\
\hline PYR & R5 & 0 & 0.1 & 97 & 2,893 & 0.4 & 0.4 & 93 & 2,086 & 0.1 & 0.3 & 30 & 1,154 & $\ldots$ & $\ldots$ & $\ldots$ & $\ldots$ & $\ldots$ & $\ldots$ & $\ldots$ & $\ldots$ & $\ldots$ & $\ldots$ & $\ldots$ & .. \\
\hline PYR & R6 & & & & & & & & & & & & & & & & & & & & & & & & \\
\hline TEB & R3 & & & & & & & & & & & & & & & & & & & & & & & & \\
\hline PYR & R5 & $\ldots$ & $\ldots$ & $\ldots$ & $\ldots$ & $\ldots$ & $\ldots$ & $\ldots$ & $\ldots$ & $\ldots$ & $\ldots$ & $\ldots$ & $\ldots$ & 0.9 & 1.0 & 86 & 2,692 & 0 & 0.0 & 86 & $\ldots$ & 0.0 & 2.6 & 84 & 1,675 \\
\hline TEB & R6 & & & & & & & & & & & & & & & & & & & & & & & & \\
\hline $\operatorname{LSD}(P$ & $=0.05)$ & ns & 0.5 & 5 & ns & 1.0 & 2.4 & 18 & 329 & 0.2 & ns & 39 & $\mathrm{~ns}$ & ns & 3.0 & 19 & 252 & $\mathrm{~ns}$ & 0.2 & 39 & $\ldots$ & ns & 5.9 & 27 & ns \\
\hline
\end{tabular}

a Fungicides tested at each location: NTC = nontreated control, PYR = pyraclostrobin (Headline, BASF), and TEB = tebuconazole $($ Tebuzol, United Phosphorus Inc.).

b Timing of fungicide application according to sunflower growth stages defined by Schneiter et al. (1998).

c $\%$ Sev $=$ mean severity on upper four leaves of sunflower using assessment diagrams (Friskop et al. 2011a).

$\mathrm{d} \%$ Disease control $=[(1-(\mathrm{rAUDPC}$ of a treatment $/ \mathrm{rAUDPC}$ of the NTC $)) \times 100] ;$ where $\mathrm{rAUDPC}=\mathrm{AUDPC} /\left(t_{f}-t_{0}\right) \times 100, \mathrm{AUDPC}=\sum_{i=1}^{n}\left(\left(y_{i}+y_{i+1} / 2\right)\left(t_{i+1}-t_{i}\right)\right)$ where $y_{i}=$ rust severity at the $i$-th observation, $t_{i}=$ time (days) at the $i$-th observation, and $n=$ total number of observations, adapted from Madden et al. (2007).

e Yield was not obtained at GRAN 2009 due to high winds and severe lodging prior to harvest. 
Table 4. Sunflower rust severity, percent disease control, and yield for early onset timing trials in 2010 and 2011

\begin{tabular}{|c|c|c|c|c|c|c|c|c|c|c|c|c|c|c|c|c|c|c|c|c|c|}
\hline & & \multicolumn{4}{|c|}{ CREC 2010} & \multicolumn{4}{|c|}{ GRAN 2010} & \multicolumn{4}{|c|}{ LREC 2010} & \multicolumn{4}{|c|}{ GRAN 2011} & \multicolumn{4}{|c|}{ LREC 2011} \\
\hline \multicolumn{2}{|c|}{ Treatments } & \multirow{2}{*}{$\begin{array}{l}\text { R5 } \\
\text { Sev } \\
\% \text { c }\end{array}$} & \multirow{2}{*}{$\begin{array}{c}\text { R7 } \\
\text { Sev } \\
\%\end{array}$} & \multirow{2}{*}{$\begin{array}{c}\% \\
\text { Dis } \\
\text { Cont }^{\mathrm{d}}\end{array}$} & \multirow{2}{*}{$\begin{array}{l}\text { Yld } \\
\text { (kg/ } \\
\text { ha) }\end{array}$} & \multirow{2}{*}{$\begin{array}{c}\text { R5 } \\
\text { Sev } \\
\%\end{array}$} & \multirow{2}{*}{$\begin{array}{c}\text { R7 } \\
\text { Sev } \\
\%\end{array}$} & \multirow{2}{*}{$\begin{array}{c}\% \\
\text { Dis } \\
\text { Cont }\end{array}$} & \multirow{2}{*}{$\begin{array}{l}\text { Yld } \\
\text { (kg/ } \\
\text { ha) }\end{array}$} & \multirow{2}{*}{$\begin{array}{c}\text { R5 } \\
\text { Sev } \\
\%\end{array}$} & \multirow{2}{*}{$\begin{array}{c}\text { R7 } \\
\text { Sev } \\
\%\end{array}$} & \multirow{2}{*}{$\begin{array}{c}\% \\
\text { Dis } \\
\text { Cont }\end{array}$} & \multirow{2}{*}{$\begin{array}{l}\text { Yld } \\
\text { (kg/ } \\
\text { ha) }\end{array}$} & \multirow{2}{*}{$\begin{array}{c}\text { R5 } \\
\text { Sev } \\
\%\end{array}$} & \multirow{2}{*}{$\begin{array}{c}\text { R7 } \\
\text { Sev } \\
\%\end{array}$} & \multirow{2}{*}{$\begin{array}{c}\% \\
\text { Dis } \\
\text { Cont }\end{array}$} & \multirow{2}{*}{$\begin{array}{l}\text { Yld } \\
\text { (kg/ } \\
\text { ha) }\end{array}$} & \multirow{2}{*}{$\begin{array}{c}\text { R5 } \\
\text { Sev } \\
\%\end{array}$} & \multirow{2}{*}{$\begin{array}{c}\text { R7 } \\
\text { Sev } \\
\%\end{array}$} & \multirow{2}{*}{$\begin{array}{c}\% \\
\text { Dis } \\
\text { Cont }\end{array}$} & \multirow{2}{*}{$\begin{array}{l}\text { Yld } \\
\text { (kg/ } \\
\text { ha) }\end{array}$} \\
\hline Fung ${ }^{a}$ & $\mathbf{A p p}^{\mathbf{b}}$ & & & & & & & & & & & & & & & & & & & & \\
\hline NTC & $\ldots$ & 0.5 & 7.3 & 0 & $\ldots^{\mathrm{e}}$ & 0.1 & 4.7 & 0 & $\ldots^{f}$ & 0.4 & 8.3 & 0 & 1,270 & 0.5 & 2.9 & 0 & 1,777 & 1.3 & 3.7 & 0 & 1,211 \\
\hline PYR & V8-V12 & 0.1 & 5.4 & 38 & $\ldots$ & 0.2 & 3.8 & 16 & $\ldots$ & 0.4 & 5.9 & 17 & 1,314 & 0.3 & 3.0 & 3 & 1,828 & 0.6 & 2.2 & 41 & 1,719 \\
\hline $\begin{array}{l}\text { PYR } \\
\text { PYR }\end{array}$ & $\begin{array}{l}\text { V8-V12 } \\
\text { R1 }\end{array}$ & 0.1 & 4.0 & 50 & $\ldots$ & 0.0 & 1.4 & 69 & $\ldots$ & 0.2 & 5.9 & 17 & 1,500 & 0.0 & 1.4 & 50 & 2,351 & 0.3 & 1.5 & 68 & 2,052 \\
\hline $\begin{array}{l}\text { PYR } \\
\text { PYR }\end{array}$ & $\begin{array}{l}\text { V8-V12 } \\
\text { R5 }\end{array}$ & 0.2 & 1.5 & 74 & $\ldots$ & 0.1 & 1.1 & 71 & $\cdots$ & 0.3 & 4.3 & 40 & 1,734 & 0.1 & 0.7 & 65 & 2,120 & 1.0 & 1.6 & 38 & 1,534 \\
\hline PYR & V8-V12 & & & & & & & & & & & & & & & & & & & & \\
\hline PYR & $\mathrm{R} 1$ & 0.0 & 1.6 & 76 & $\ldots$ & 0.0 & 0.7 & 85 & $\ldots$ & 0.2 & 2.9 & 58 & 1,675 & 0.0 & 0.7 & 77 & 2,624 & 0.2 & 0.6 & 82 & 2,455 \\
\hline PYR & R5 & & & & & & & & & & & & & & & & & & & & \\
\hline PYR & $\mathrm{R} 1$ & 0.1 & 3.4 & 56 & $\ldots$ & 0.0 & 3.4 & 24 & $\ldots$ & 0.3 & 6.4 & 23 & 1,505 & 0.1 & 2.0 & 33 & 2,108 & 0.5 & 2.4 & 42 & 1,866 \\
\hline $\begin{array}{l}\text { PYR } \\
\text { PYR }\end{array}$ & $\begin{array}{l}\text { R1 } \\
\text { R5 }\end{array}$ & 0.1 & 1.3 & 75 & $\ldots$ & 0.0 & 0.7 & 85 & $\ldots$ & 0.5 & 5.1 & 19 & 1,470 & 0.0 & 0.7 & 71 & 2,521 & 0.4 & 0.9 & 72 & 2,389 \\
\hline PYR & $\mathrm{R} 5$ & 0.3 & 2.2 & 57 & $\cdots$ & 0.2 & 0.9 & 72 & $\ldots$ & 0.4 & 6.7 & 12 & 1,055 & 0.1 & 1.0 & 59 & 1,681 & 1.4 & 1.7 & 29 & 1,319 \\
\hline TEB & V8-V12 & $\ldots$ & $\cdots$ & $\ldots$ & $\ldots$ & 0.1 & 4.6 & 0.5 & $\cdots$ & $\ldots$ & $\ldots$ & $\ldots$ & $\ldots$ & 0.2 & 1.7 & 26 & 1,890 & $\ldots$ & $\cdots$ & $\cdots$ & $\ldots$ \\
\hline $\begin{array}{l}\text { TEB } \\
\text { TEB }\end{array}$ & $\begin{array}{l}\text { V8-V12 } \\
\text { R1 }\end{array}$ & $\cdots$ & $\cdots$ & $\cdots$ & $\cdots$ & 0.0 & 0.8 & 83 & $\ldots$ & $\ldots$ & $\cdots$ & $\ldots$ & $\ldots$ & 0.0 & 0.9 & 63 & 1,991 & $\ldots$ & $\ldots$ & $\cdots$ & $\cdots$ \\
\hline $\begin{array}{l}\text { TEB } \\
\text { TEB }\end{array}$ & $\begin{array}{l}\text { V8-V12 } \\
\text { R5 }\end{array}$ & $\cdots$ & $\cdots$ & $\cdots$ & $\cdots$ & 0.1 & 0.9 & 79 & $\ldots$ & $\cdots$ & $\cdots$ & $\cdots$ & $\cdots$ & 0.3 & 0.5 & 60 & 2,229 & $\ldots$ & $\cdots$ & $\cdots$ & $\cdots$ \\
\hline TEB & V8-V12 & & & & & & & & & & & & & & & & & & & & \\
\hline TEB & $\mathrm{R} 1$ & $\ldots$ & $\ldots$ & $\ldots$ & $\ldots$ & 0.0 & 0.5 & 90 & $\ldots$ & $\ldots$ & $\ldots$ & $\ldots$ & $\ldots$ & 0.0 & 0.5 & 82 & 2,197 & $\ldots$ & $\ldots$ & $\ldots$ & $\ldots$ \\
\hline TEB & R5 & & & & & & & & & & & & & & & & & & & & \\
\hline TEB & $\mathrm{R} 1$ & $\ldots$ & $\ldots$ & $\ldots$ & $\ldots$ & 0.0 & 0.6 & 88 & $\ldots$ & $\ldots$ & $\ldots$ & $\ldots$ & $\ldots$ & 0.1 & 0.9 & 57 & 2,067 & $\ldots$ & $\ldots$ & $\ldots$ & $\ldots$ \\
\hline $\begin{array}{l}\text { TEB } \\
\text { TEB }\end{array}$ & $\begin{array}{l}\text { R1 } \\
\text { R5 }\end{array}$ & . & .. & $\cdots$ & $\cdots$ & 0.0 & 0.6 & 88 & $\ldots$ & $\ldots$ & . & .. & $\ldots$ & 0.1 & 0.4 & 78 & 2,235 & $\ldots$ & . & $\ldots$ & $\ldots$ \\
\hline TEB & R5 & $\ldots$ & $\ldots$ & $\ldots$ & $\ldots$ & 0.2 & 1.0 & 73 & $\ldots$ & $\ldots$ & $\ldots$ & $\ldots$ & $\ldots$ & 0.5 & 0.6 & 42 & 1,994 & $\ldots$ & $\ldots$ & $\ldots$ & $\ldots$ \\
\hline LSD $(I$ & $=0.05)$ & 0.3 & 2.2 & 20 & $\ldots$ & 0.1 & 1.2 & 25 & $\ldots$ & 0.2 & 1.4 & 18 & 343 & 0.2 & 1.0 & 23 & 411 & 0.7 & 0.8 & 19 & 525 \\
\hline
\end{tabular}

a Fungicides tested at each location: NTC = nontreated control, PYR = pyraclostrobin (Headline, BASF), and TEB = tebuconazole (Tebuzol, United Phosphorus Inc.).

b Timing of fungicide application according to sunflower growth stages defined by Schneiter et al. (1998).

c $\% \mathrm{Sev}=$ mean severity on upper four leaves of sunflower using assessment diagrams (Friskop et al. 2011a)

$\mathrm{d} \%$ Disease control $=[(1-(\mathrm{rAUDPC}$ of a treatment $/ \mathrm{rAUDPC}$ of the NTC $)) \times 100] ;$ where $\mathrm{rAUDPC}=\mathrm{AUDPC} /\left(t_{f}-t_{0}\right) \times 100, \mathrm{AUDPC}=\sum_{i=1}^{n}\left(\left(y_{i}+y_{i+1} / 2\right) \times\right.$ $\left.\left(t_{i+1}-t_{i}\right)\right)$ where $y_{i}=$ rust severity at the $i$-th observation, $t_{i}=$ time (days) at the $i$-th observation, and $n=$ total number of observations, as adapted from Madden et al. (2007).

e Yield was not obtained at CREC 2010 due to a severe sunflower midge infestation.

f Yield was not obtained at GRAN 2010 due to high winds and severe lodging prior to harvest.

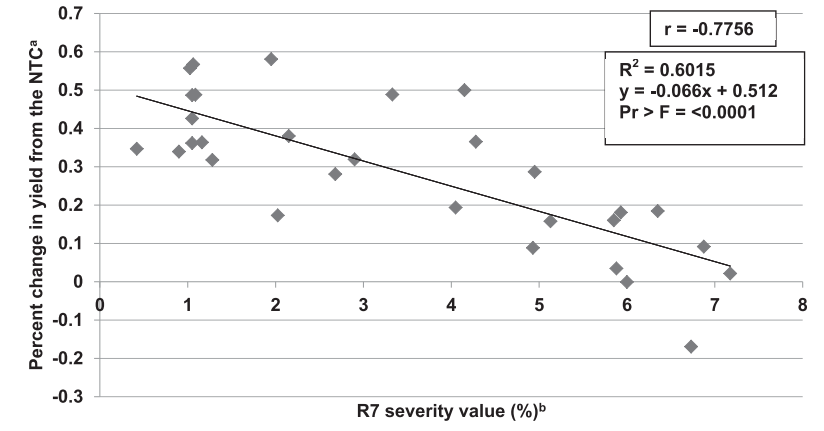

a Percent change in yield from NTC $=$ (fungicide treatment mean yield - NTC mean yield $) /$ NTC mean yield ${ }^{\mathrm{b}} \mathrm{R} 7$ severity value $=$ mean rust severity on upper four leaves according to rust assessment diagrams (Friskop et al. 2011a) during R7 growth stage (Schneiter et al. 1998).

Fig. 2. Sunflower yield loss assessment correlating R7 disease severity values and percent change in yield from the nontreated control at CREC 2008 timing, CREC 2009 efficacy, CREC 2009 timing, and LREC 2010. a severity value of $1 \%$ or less, was more efficacious for reduction of disease severity than any other timing investigated in this study.

In most trials, levels of disease control were statistically the same among QoI and DMI fungicide treatments. Few differences in disease control among the three fungicides labeled for sunflower rust in the United States (azoxystrobin, pyraclostrobin, and tebuconazole) at the time of the study were observed. However, when differences were observed, disease control from tebuconazole applications was higher than pyraclostrobin or azoxystrobin applications. Fungicides not labeled for sunflowers generally controlled rust as well as labeled fungicides in their respective FRAC groups.

Fungicide timing proved to be a critical component in disease management. Of all single timings evaluated throughout this study, disease reductions appeared most frequently with a single application at R5. Applications at earlier or later growth stages tended to control disease less than applications made at R5. This could be because applications made earlier than R5 may have not provided protection to the top leaves prior to leaf expansion, or that the duration of 
protection was inadequate for the length of growing season remaining. Conversely, an application later than R5 may have had a more limited impact on disease control because a high severity had been reached by the time of application. In four trials, disease control from a single R5 fungicide application reduced rust as much as three applications (CREC 2008-Normal Onset, CREC 2009-Normal Onset, GRAN 2009-Normal Onset, and LREC 2009-Normal Onset). With the exception of GRAN 2009, it is notable that these trials had the highest R7 rust severities of the six 2008 and 2009 timing trials. This indicates that reduction of rust with a single application was as effective as three applications, under the highest disease pressure situations observed in this study.

Although one objective of the research project was to evaluate fungicide application timing in an "early onset" disease scenario in 2010 and 2011, rust did not develop early enough to simulate an early onset epidemic. However, some trends were noticed. Based on disease control values, single applications made at V8-V12 did not provide satisfactory disease control and multiple applications of a fungicide that included an R5 application often were among the highest for disease control. Prophylactic fungicide applications for yield gain independent of disease control have been suggested on sunflower at the R1 growth stage. Trials established in 2010 and 2011 included a R1 application, but no correlation between single R1 applications and yield was observed.

Yield loss correlations with sunflower rust severity are very limited in the literature. This is largely because sunflowers are sensitive to numerous yield-limiting and unmanageable problems such as bird damage, insects, other diseases, and high winds. Similar stresses were observed in our study; for example, sunflower midge caused a total yield loss at CREC 2010, Phomopsis stem canker reduced yields by 50\% in LREC 2011, and straight-line winds (estimated to be between 26.8 and $33.5 \mathrm{~ms}^{-1}$ ) caused $100 \%$ lodging at GRAN 2010. Consequently, yield loss data under sunflower rust pressure in the literature is limited. Of the 17 trials in our study, eight trials had rust pressure in excess of 5\% at R7, four of which had statistical differences yield differences that were believed to be primarily or solely due to rust. In these trials, a negative correlation ( $\mathrm{r}=-0.7756)$ was observed between $\mathrm{R} 7 \mathrm{se}-$ verity and percent change in yield, resulting in a yield reduction of $6.6 \%$ for every $1 \%$ increase in rust severity.

Based on previous work, an action threshold of $3 \%$ severity was recommended to reduce disease pressure and limit yield loss (Shtienberg 1995). Data from these studies indicate that an application at a lower disease severity is more appropriate, but choice of DMI or QoI product infrequently matters. As a result of these studies, a threshold of $1 \%$ disease severity on the upper four leaves, when occurring at or before R5, has been suggested and widely accepted in the United States by the sunflower industry and producers in North Dakota.

\section{Acknowledgments}

We thank staff members at NDSU's Carrington research extension center, NDSU's Langdon research extension center, and CHS for their contributions to this project. We thank BASF, Bayer CropScience, Dupont Agricultural Products, Syngenta Crop Protection, United Phosphorous Inc., and Valent USA for chemical donation and technical support. We thank the National Sunflower Association, the North Dakota State Board of Agricultural Research and Education, the North Dakota Department of Agriculture, and the NDSU Agricultural Experiment station for funding.

\section{Literature Cited}

Berglund, D. 2007. Introduction. In: Sunflower Production Guide. D. Berglund, ed. North Dakota State University Cooperative Extension Service Publication. A-1331.

Friskop, A., Markell, S., Gulya, T., Halley, S., Schatz, B., Schaefer, J., Wunsch, M., Meyer, S., Kandel, H., Acevedo, M., Venette, J., and Venette, R. 2011a. Sunflower rust. North Dakota State University Cooperative Extension Service Publication. PP-1557.

Friskop, A. J., Schatz, B., Mathew, F., Hendrickson, P., Gulya, T., and Markell, S. 2011 b. Sunflower hybrid susceptibility and the impact on yield to sunflower rust in North Dakota. Phytopathology 101:S2.2.

Gulya, T., Rashid, K. Y., and Masirevic, S. M. 1997. Sunflower diseases. Pages 263-379 in: Sunflower Technology and Production. A. A. Schneiter, ed. American Society Agronomy, Madison, WI

Gulya, T. J., Venette, R., Venette, J. R., and Lamey, H. A. 1990. Sunflower rust. North Dakota State University Cooperative Extension Service Publication. PP-998.

Harveson, R. 2010. First report of early spore stages of sunflower rust, caused by Puccinia helianthi, in Nebraska. Plant Health Prog. doi: 10.1094/PHP-2010 0315-01-BR.

Kandel, H. 2012. 2011 National Sunflower Association survey. Proc. 34th Sunflower Research Workshop. National Sunflower Association, Bismarck, ND. Available at http://www.sunflowernsa.com/uploads/research/1140/2011. sunflower.survey.updated_kandel_12.pdf

Madden, L. V., Hughes, G., and van den Bosch, F. 2007. The Study of Plant Disease Epidemics. American Phytopathological Society, St. Paul, MN.

Markell, S. G., Griffey, C. A., and Milus, E. A. 2009a. Inheritance of resistance to stripe rust in three lines of soft red winter wheat. Crop Sci. 49:521-528.

Markell, S., Gulya, T., McKay, K., Hutter, M., Hollingsworth, C., Ulstad, V., Koch, R., and Knudsvig, A. 2009b. Widespread occurrence of the aecial stage of sunflower rust caused by Puccinia helianthi in North Dakota and Minnesota in 2008. Plant Dis. 93:668.

McMullen, M. P., and Markell, S. G. 2010. 2011 North Dakota field crop fungicide guide. North Dakota State University Cooperative Extension Service Publication. PP-622.

Mueller, T. A., Miles, M. R., Morel, W., Marois, J. J., Wright, D. L., Kemerait R. C., Levy, C., and Hartman, G. L. 2009. Effect of fungicide and timing of application on soybean rust severity and yield. Plant Dis. 93:243-248

Olson, L., Halley, S., Wunsch, M., Schatz, B., Meyer, S., Goswami, R., Mathew, F., Lamppa, R., Hendrickson, P., and Markell, S. 2011. Evaluation of fungicide efficacy and timing from management of common bean rust in North Dakota. Phytopathology 101:S2.2-2.3.

Peterson, R. F., Campbell, A. B., and Hannah, A. E. 1948. A diagrammatic scale for estimating rust intensity on leaves and stems of cereals. Can. J. Res. 26c: 496-500.

Schneiter, A. A., Miller, J. F., and Berglund, D. R. 1998. Stages of sunflower development. North Dakota State University Extension Publication. A-1145.

Shtienberg, D. 1995. Rational suppression of sunflower rust: development and evaluation of an action threshold. Plant Dis. 79:506-510.

Singh, J. P. 1975. Field evaluation of fungicides for the control of sunflower rust in Kenya. Plant Dis. Rep. 59:200-202.

Thakore, B. B., Mathur, S., Chakravarti, D. P., Singh, R. B., and Singh, R. D. 1980 Increase in sunflower yield by controlling rust with systemic and non-systemic fungicides. J. Turk. Phytopathol. 9:89-96.

Wanyera, R., Macharia, J. K., Kilonzo, S. M., and Kamundia, J. W. 2009. Foliar fungicides to control wheat stem rust, race TTKS (Ug99), in Kenya. Plant Dis. 93:929-932. 